\title{
The line-of-sight towards GRB 030429 at $z=2.66$ : Probing the matter at stellar, galactic and intergalactic scales ${ }^{\star}$
}

\author{
P. Jakobsson ${ }^{1,2}$, J. Hjorth ${ }^{1}$, J. P. U. Fynbo ${ }^{1,3}$, M. Weidinger ${ }^{3,4}$, J. Gorosabel ${ }^{5,6}$, C. Ledoux ${ }^{7}$, \\ D. Watson ${ }^{1}$, G. Björnsson ${ }^{2}$, E. H. Gudmundsson ${ }^{2}$, R. A. M. J. Wijers ${ }^{8}$, P. Møller ${ }^{4}$, K. Pedersen ${ }^{1}$, \\ J. Sollerman ${ }^{9}$, A. A. Henden ${ }^{10}$, B. L. Jensen ${ }^{1}$, A. Gilmore ${ }^{11}$, P. Kilmartin ${ }^{11}$, A. Levan ${ }^{12}$, \\ J. M. Castro Cerón ${ }^{6}$, A. J. Castro-Tirado ${ }^{5}$, A. Fruchter ${ }^{6}$, C. Kouveliotou ${ }^{13}$, N. Masetti ${ }^{14}$, and N. Tanvir ${ }^{15}$ \\ 1 Niels Bohr Institute, Astronomical Observatory, University of Copenhagen, Juliane Maries Vej 30, 2100 Copenhagen, \\ Denmark \\ e-mail: pallja@astro.ku.dk \\ 2 Science Institute, University of Iceland, Dunhaga 3, 107 Reykjavík, Iceland \\ 3 Department of Physics and Astronomy, University of Aarhus, Ny Munkegade, 8000 Århus C, Denmark \\ ${ }^{4}$ European Southern Observatory, Karl-Schwarzschild-Straße 2, 85748, Garching bei München, Germany \\ 5 Instituto de Astrofísica de Andalucía (IAA-CSIC), PO Box 03004, 18080 Granada, Spain \\ 6 Space Telescope Science Institute, 3700 San Martin Drive, Baltimore, MD 21218, USA \\ 7 European Southern Observatory, Alonso de Córdova 3107, Casilla 19001, Santiago 19, Chile \\ 8 Astronomical Institute, University of Amsterdam, Kruislaan 403, 1098 SJ Amsterdam, The Netherlands \\ 9 Stockholm Observatory, Department of Astronomy, AlbaNova, 10691 Stockholm, Sweden \\ 10 USRA/USNO Flagstaff Station, PO Box 1149, Flagstaff, AZ 86002, USA \\ 11 Mt John Observatory, Department of Physics and Astronomy, University of Canterbury, Christchurch 8020, New Zealand \\ 12 Department of Physics and Astronomy, University of Leicester, University Road, Leicester, LE1 7RH, UK \\ 13 NASA MSFC, SD-50 Huntsville, AL 35812, USA \\ 14 Istituto di Astrofisica Spaziale e Fisica Cosmica, Sezione di Bologna, CNR, Via Gobetti 101, 40129 Bologna, Italy \\ 15 Department of Physical Sciences, University of Hertfordshire, College Lane, Hatfield, Herts AL10 9AB, UK
}

Received 5 May 2004 / Accepted 20 July 2004

\begin{abstract}
We report the discovery of the optical afterglow (OA) of the long-duration gamma-ray burst GRB 030429, and present a comprehensive optical/near-infrared dataset used to probe the matter at different distance scales, i.e. in the burst environment, in the host galaxy and in an intervening absorber. A break in the afterglow light curve is seen approximately 1 day from the onset of the burst. The light curve displays a significant deviation from a simple broken power-law with a bright 1.5 mag bump with a duration of 2-3 days. The optical/near-infrared spectral energy distribution is best fit with a power-law with index $\beta=-0.36 \pm 0.12$ reddened by an SMC-like extinction law with (a modest) $A_{V}=0.34 \pm 0.04$. In addition, we present deep spectroscopic observations obtained with the Very Large Telescope. The redshift measured via metal absorption lines in the OA is $z=2.658 \pm 0.004$. Based on the damped Ly $\alpha$ absorption line in the OA spectrum we measure the H I column density to be $\log N(\mathrm{HI})=21.6 \pm 0.2$. This confirms the trend that GRBs tend to be located behind very large H I column densities. The resulting dust-to-gas ratio is consistent with that found in the SMC, indicating a low metallicity and/or a low dust-to-metal ratio in the burst environment. We find that a neighbouring galaxy, at a separation of only $11^{\prime \prime} 2$, has $z=0.841 \pm 0.001$, ruling it out as the host of GRB 030429. The small impact parameter of this nearby galaxy, which is responsible for Mg II absorption in the OA spectrum, is in contrast to previous identifications of most QSO absorption-selected galaxy counterparts. Finally, we demonstrate that the OA was not affected by strong gravitational lensing via the nearby galaxy.
\end{abstract}

Key words. gamma rays: bursts - galaxies: distances and redshifts - galaxies: high-redshift - ISM: dust, extinction quasars: absorption lines

\section{Introduction}

Considerable progress has been made in the understanding of gamma-ray bursts (GRBs) since BeppoSAX started

* Based on observations made with ESO Telescopes at the Paranal Observatory by GRACE under programme ID 71.D-0355(A+B+C). distributing localisations with arcmin precision and van Paradijs et al. (1997) discovered the first optical afterglow (OA). Of the approximately 50 OAs that have been detected since 1997, 39 have had their redshift spectroscopically determined. As shown in Fig. 1, the mean redshift is around 1.3 with 13 GRBs at $z>1.5$. 


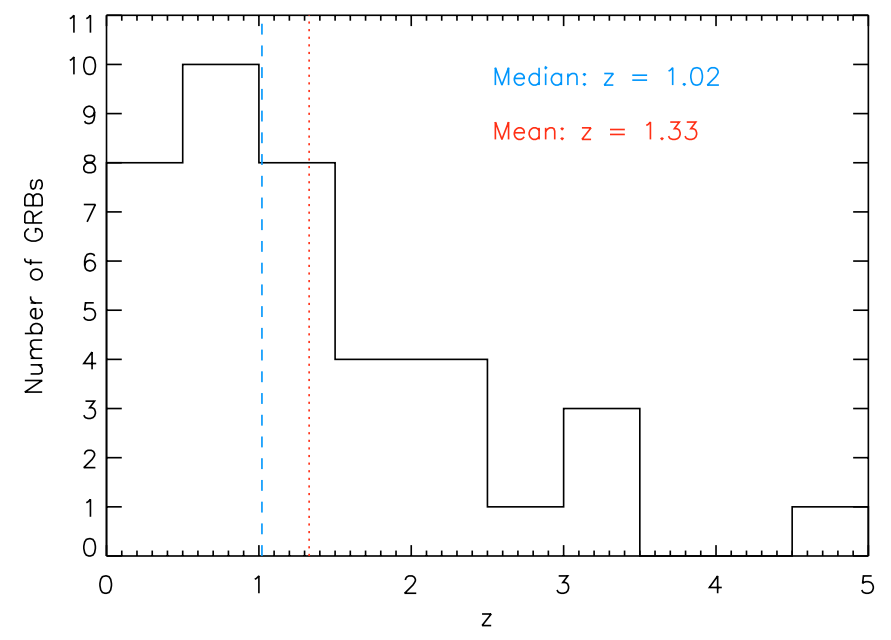

Fig. 1. A histogram showing the distribution of GRB redshifts known to date (October 2004). The median value of the 39 redshifts is indicated by the dashed line, while the mean value is shown with the dotted line.

Independently of the brightness of GRB host galaxies, OAs can be used as tools to obtain information about the gas, metallicity and dust content of the host. In particular, the optical/near-infrared (NIR) spectral energy distribution (SED) can provide the extinction, while the neutral hydrogen column density, $N(\mathrm{HI})$, can be derived from the damped Ly $\alpha$ absorption spectroscopy, provided that the burst is distant enough for the Ly $\alpha$ line to be redshifted into the optical/near-UV domain. The detection of damped Ly $\alpha$ absorption lines in the spectra of several GRB afterglows (Jensen et al. 2001; Fynbo et al. 2001b; Hjorth et al. 2003a; Vreeswijk et al. 2004) is consistent with the now firmly established link between long-duration GRBs and core-collapse of massive stars (e.g. Hjorth et al. 2003b; Stanek et al. 2003).

Like QSOs, GRB afterglows are distant sources that can be used as cosmological beacons to investigate intervening galaxies unrelated to the burst itself. For the current discussion, the most important difference between these two astronomical phenomena is that OAs are ephemeral, vanishing altogether within a couple of months. This leaves the line-of-sight clear and without any interference from a bright object. Another significant difference is that afterglows typically have featureless spectra, making it easier to identify intervening absorption systems.

In this paper we present the detection of the OA of GRB 030429 and use it to probe the progenitor environment (sub-pc scale), the host galaxy properties (kpc scale) and an intervening $\mathrm{Mg}$ II absorber (Gpc scale). The organisation of the paper is as follows. The optical, NIR and spectroscopic observations are presented in Sect. 2. In Sect. 3 we investigate the optical/NIR light curve and the SED. We analyze the spectrum and give the redshift of GRB 030429 in Sect. 4. In addition we fit a H I column density model to a damped Ly $\alpha$ line present in the OA spectrum. In Sect. 5 we use the derived properties of the OA to compare our results with afterglow models. We also discuss the properties of the nearby $\mathrm{Mg}$ II absorbing galaxy and assess whether it could be responsible for strong gravitational lensing of the OA. Finally, the main results are summarised in Sect. 6.
We adopt a cosmology where the Hubble parameter is $H_{0}=$ $70 \mathrm{~km} \mathrm{~s}^{-1} \mathrm{Mpc}^{-1}, \Omega_{\mathrm{m}}=0.3$ and $\Omega_{\Lambda}=0.7$. For these parameters, a redshift of $2.66(0.84)$ corresponds to a luminosity distance of $21.99 \mathrm{Gpc}(5.33 \mathrm{Gpc})$ and a distance modulus of 46.7 (43.6). One arcsecond is equivalent to 7.96 (7.63) proper kiloparsecs, and the lookback time is $11.1 \mathrm{Gyr}(7.0 \mathrm{Gyr})$.

\section{Observations}

GRB 030429 was detected by the French Gamma Telescope (FREGATE), Wide Field X-ray Monitor (WXM) and Soft X-ray Camera (SXC) on-board the HETE-2 satellite on 2003 April 29.446 UT. The burst had a duration of $10.3 \mathrm{~s}$, placing it in the "long-duration" burst category. Initially, a 1' radius error circle was circulated via the GRB Coordinate Network $(\mathrm{GCN})^{1}$ approximately $2 \mathrm{~h}$ after the burst. A week later the correct error radius of $2^{\prime}$ was distributed (Doty et al. 2003).

The OA was discovered at the Mt. John 0.6-m telescope $\sim 3.5 \mathrm{~h}$ after the burst (Gilmore et al. 2003). It was identified outside the initial erroneous SXC 1' error circle after comparison with a DSS-2 red plate. The OA was monitored in the optical during the following days with the $1.0-\mathrm{m}$ and $1.55-\mathrm{m}$ telescopes at the US Naval Observatory Flagstaff Station (NOFS), and with the Very Large Telescope (VLT), using either the Antu/FORS1 or the Yepun/FORS2 combination (Fynbo et al. 2003a). In addition, Antu/ISAAC was used to obtain NIR images of the OA shortly after the burst. A VLT/FORS2 image of the OA and its surrounding field is displayed in Fig. 2. The journal of our observations is given in Table 1 .

Using Antu we obtained $11 \times 600 \mathrm{~s}$ spectra of the OA starting on 2003 May 2.214 UT, 2.77 days after the burst (Weidinger et al. 2003a,b). The data were acquired with the FORS1 instrument in long-slit spectroscopy mode with the G300V grism, GG375 order separation filter and a 1".3 wide slit. This resulted in a wavelength coverage from 3900-8000 ̊. The slit was oriented with a position angle of -71.1 in order to cover both the OA and a neighbouring galaxy (lower panel of Fig. 2). The observations were done with the standard resolution collimator. The individual spectra were combined, yielding a seeing of 0 . $^{\prime} 8$ and a spectral resolution of $10 \AA$ FWHM. The projected pixel size was $0.2 \times 2.58 \AA$.

Berger \& Frail (2003) observed the error circle of GRB 030429 with the Very Large Array at $8.46 \mathrm{GHz}, 2.65$, 5.63 and 16.68 days after the burst. No radio sources were detected at the position of the OA down to a $3 \sigma$ limit of $0.18,0.16$ and $0.10 \mathrm{mJy}$, respectively.

\section{Imaging}

\subsection{Photometry}

The data were reduced using standard techniques for de-biasing and flat-fielding. The photometry of the afterglow was carried out using point-spread function (PSF) fitting photometry (Stetson 1987, 1994). For the VLT images, the relative optical magnitudes were transformed to the Johnson photometric system using observations of Landolt (1992) standards. For the

\footnotetext{
${ }^{1}$ http://gcn.gsfc.nasa.gov/gcn/
} 
Table 1. A log of the GRB 030429 follow-up imaging observations. Upper limits are $2 \sigma$ in a circular aperture with radius $1^{\prime \prime}$. No correction for Galactic extinction has been applied to the photometry.

\begin{tabular}{|c|c|c|c|c|}
\hline $\begin{array}{c}\Delta t^{a} \\
\text { [days] }\end{array}$ & $\begin{array}{l}\text { Telescope/ } \\
\text { Instrument }\end{array}$ & Magnitude $^{b}$ & $\begin{array}{c}\text { Seeing } \\
{[\operatorname{arcsec}]}\end{array}$ & $\begin{array}{c}\text { Exp. time } \\
\text { [s] }\end{array}$ \\
\hline \multicolumn{5}{|l|}{$B$-band: } \\
\hline 1.747 & 1.0-m NOFS & $22.24 \pm 0.30$ & 2.3 & 720 \\
\hline \multicolumn{5}{|l|}{$V$-band: } \\
\hline 0.535 & Yepun/FORS2 & $21.45 \pm 0.03$ & 0.7 & $5 \times 180$ \\
\hline 0.764 & Yepun/FORS2 & $21.79 \pm 0.03$ & 0.8 & $5 \times 180$ \\
\hline 1.755 & 1.0-m NOFS & $21.89 \pm 0.32$ & 2.4 & 480 \\
\hline 2.541 & Yepun/FORS2 & $23.18 \pm 0.11$ & 0.9 & $5 \times 180$ \\
\hline 6.631 & Yepun/FORS2 & $>26.0$ & 1.3 & $5 \times 180$ \\
\hline \multicolumn{5}{|l|}{$R$-band: } \\
\hline 0.145 & 0.6-m Mt. John & $19.67 \pm 0.11$ & 3.4 & $10 \times 60$ \\
\hline 0.154 & 0.6-m Mt. John & $19.39 \pm 0.08$ & 3.3 & $10 \times 60$ \\
\hline 0.170 & 0.6-m Mt. John & $19.62 \pm 0.09$ & 3.3 & $10 \times 60$ \\
\hline 0.214 & 0.6-m Mt. John & $19.56 \pm 0.11$ & 4.8 & $10 \times 60$ \\
\hline 0.548 & Yepun/FORS2 & $20.86 \pm 0.04$ & 0.7 & $5 \times 180$ \\
\hline 0.777 & Yepun/FORS2 & $21.13 \pm 0.04$ & 0.8 & $5 \times 180$ \\
\hline 1.761 & 1.0-m NOFS & $21.42 \pm 0.27$ & 2.2 & 420 \\
\hline 1.862 & 1.55-m NOFS & $21.27 \pm 0.09$ & 1.2 & 600 \\
\hline 1.884 & $1.55-\mathrm{m}$ NOFS & $21.58 \pm 0.15$ & 1.4 & 600 \\
\hline 2.553 & Yepun/FORS2 & $22.54 \pm 0.06$ & 0.9 & $5 \times 180$ \\
\hline 2.793 & 1.55-m NOFS & $22.55 \pm 0.09$ & 1.3 & $5 \times 600$ \\
\hline 3.632 & Yepun/FORS2 & $23.71 \pm 0.12$ & 0.5 & $5 \times 180$ \\
\hline 6.644 & Yepun/FORS2 & $25.20 \pm 0.30$ & 1.2 & $5 \times 180$ \\
\hline 67.641 & Antu/FORS1 & $>26.3$ & 0.7 & $15 \times 180$ \\
\hline \multicolumn{5}{|l|}{ I-band: } \\
\hline 0.561 & Yepun/FORS2 & $20.29 \pm 0.05$ & 0.7 & $5 \times 180$ \\
\hline 0.790 & Yepun/FORS2 & $20.63 \pm 0.05$ & 0.8 & $5 \times 180$ \\
\hline 1.766 & 1.0-m NOFS & $20.51 \pm 0.37$ & 2.2 & 420 \\
\hline 2.566 & Yepun/FORS2 & $21.72 \pm 0.09$ & 0.6 & $5 \times 180$ \\
\hline 6.658 & Yepun/FORS2 & $24.70 \pm 0.30$ & 1.1 & $5 \times 180$ \\
\hline \multicolumn{5}{|l|}{$J_{\mathrm{s}}$-band: } \\
\hline 0.538 & Antu/ISAAC & $19.25 \pm 0.04$ & 0.6 & $20 \times 90$ \\
\hline 0.753 & Antu/ISAAC & $19.51 \pm 0.04$ & 0.7 & $20 \times 90$ \\
\hline \multicolumn{5}{|l|}{$K_{\mathrm{s}}$-band: } \\
\hline 0.568 & Antu/ISAAC & $17.70 \pm 0.06$ & 0.6 & $30 \times 60$ \\
\hline 0.783 & Antu/ISAAC & $18.01 \pm 0.06$ & 0.6 & $30 \times 60$ \\
\hline
\end{tabular}

${ }^{a}$ Days after 2003 April 29.446.

${ }^{b}$ The $0.6-\mathrm{m}$ observations were unfiltered but tied to the $R$-band as described in the text.

other telescopes we used the calibrated magnitudes of stars in the field (Henden 2003). We note that the 0.6-m observations were unfiltered, but we tied them to the $R$-band using Henden (2003) standards with similar colours as the OA. For the NIR data we used 2MASS stars in the field to transform our observations to the standard system.

As seen in Table 1 the afterglow was observed at the first two VLT epochs ( $\Delta t \approx 0.55$ days and $\Delta t \approx 0.78$ days) in all

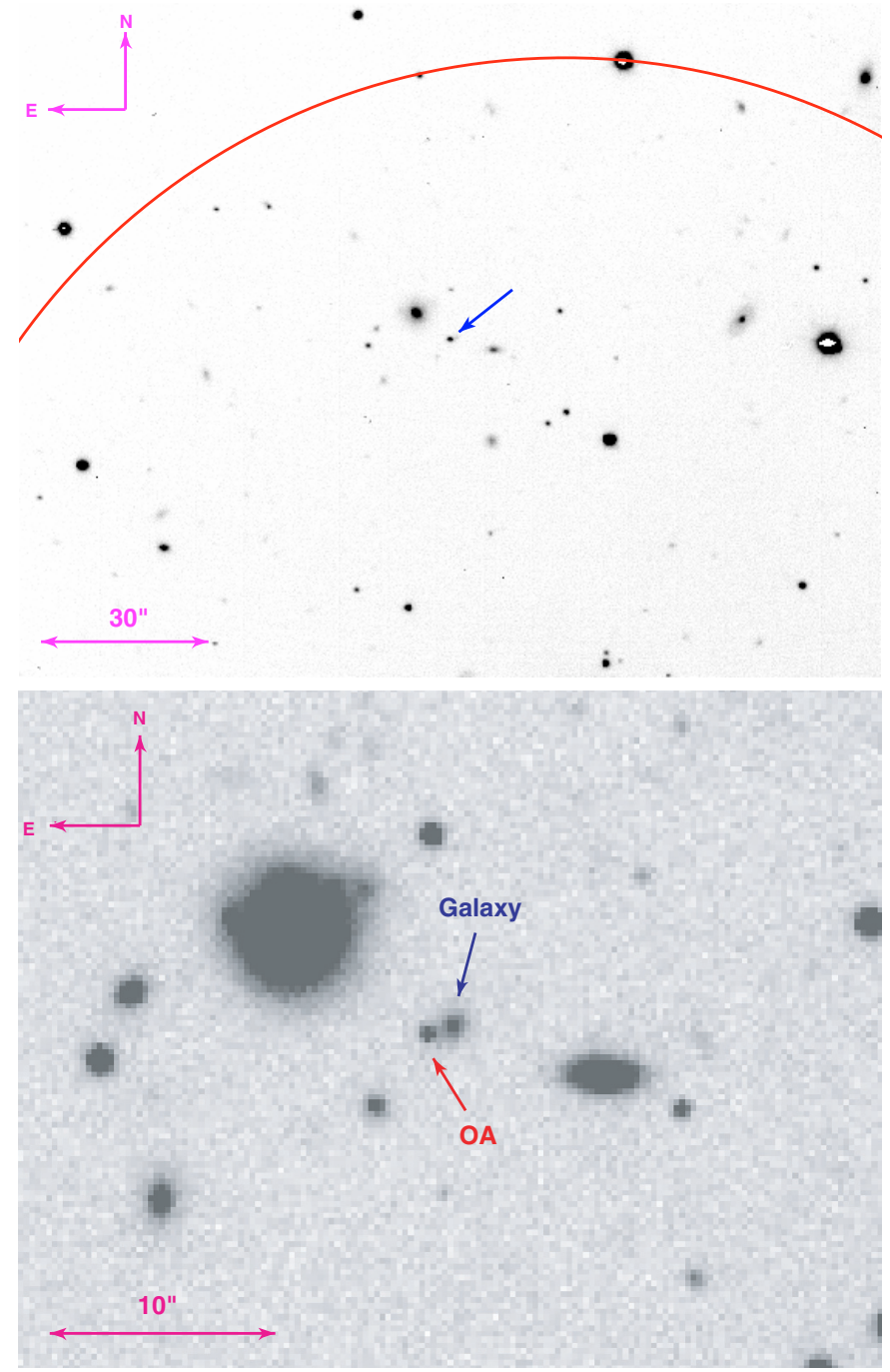

Fig. 2. Top: a 180 s $R$-band VLT/FORS2 image of the GRB 030429 optical afterglow, obtained $\sim 13 \mathrm{~h}$ after the burst occurred. The position of the optical afterglow is marked with an arrow. The SXC localization is shown with a continuous circle of radius $2^{\prime}$. Bottom: a combined $900 \mathrm{~s} R$-band VLT/FORS2 image with a seeing of 0 .' 5 , taken $\sim 3.6$ days from the onset of the burst. The field of view is smaller than shown in the top panel in order to emphasise the surroundings of the OA. A galaxy at a distance of 1 .' 2 from the OA is clearly visible and marked with an arrow.

five filters $\left(V R I J_{\mathrm{s}} K_{\mathrm{s}}\right)$ and in $V R I$ during the third VLT epoch ( $\Delta t \approx 2.55$ days). The final detection of the OA is in $R$ and $I$ at $\Delta t \approx 6.65$ days, where $\Delta t$ is the time from the onset of the burst. We also have an upper limit in $V$ at the same time, and a deep upper limit in $R$ roughly 2 months after the burst. We note that there is a galaxy $\sim 1^{\prime \prime} .2 \mathrm{WNW}$ of the OA, visible in the lower panel of Fig. 2, which was initially considered to be the probable host galaxy (see Sect. 4.2). There is no indication of a host directly underlying the OA; we can set an upper limit of $R>26.3(2 \sigma)$ from our last observation. For a future host search we include the accurate position of the afterglow based on USNO CCD Astrograph Catalog (UCAC2) stars in the field: $\operatorname{RA}(J 2000)=12^{\mathrm{h}} 13^{\mathrm{m}} 07^{\$} .495(10)$ and $\operatorname{Dec}(\mathrm{J} 2000)=$ $-20^{\circ} 54^{\prime} 49^{\prime \prime} 54(3)$ with the error in the last digits indicated in 
Table 2. Photometry of the galaxy J1213.1-2054.8, 1.'2 WNW of the optical afterglow. No correction for Galactic extinction has been applied to the photometry.

\begin{tabular}{ccccc}
\hline \hline$V$ & $R$ & $I$ & $J_{\mathrm{s}}$ & $K_{\mathrm{s}}$ \\
\hline $23.70 \pm 0.18$ & $22.70 \pm 0.12$ & $21.29 \pm 0.09$ & $20.50 \pm 0.11$ & $18.40 \pm 0.07$ \\
\hline
\end{tabular}

Table 3. The result of fitting the $R$-band light curve of the optical afterglow with a broken power-law. The different methods are described in Sect. 3.2. We note that $\chi_{\text {d.o.f. }}^{2}=\chi^{2} /$ degree of freedom, is the reduced $\chi^{2}$ of the fit.

\begin{tabular}{lccccc}
\hline \hline & $\chi_{\text {d.o.f. }}^{2}$ & d.o.f. & $\alpha_{1}$ & $\alpha_{2}$ & $t_{\mathrm{b}}$ [days] \\
\hline (i) & 6.15 & 9 & $-0.88 \pm 0.03$ & $-2.87 \pm 0.25$ & $2.26 \pm 0.10$ \\
(ii) & 2.33 & 6 & $-0.99 \pm 0.02$ & $-3.46 \pm 0.44$ & $2.76 \pm 0.11$ \\
(iii) & 1.96 & 4 & $-0.95 \pm 0.03$ & -1.72 (fixed) & $0.78 \pm 0.01$ \\
\hline
\end{tabular}

parentheses. The accuracy of the afterglow position in RA is worse than in declination due to the light contribution from the nearby galaxy, which is almost directly west of the afterglow.

In order to determine the properties of the nearby galaxy (hereafter referred to as J1213.1-2054.8) we used SExtractor (Bertin \& Arnouts 1996) to obtain its $V R I J_{\mathrm{s}} K_{\mathrm{s}}$ total magnitudes (mag_auto). Given that our first epoch data had a very similar seeing in all filters $\left(00^{\prime} 6-0{ }^{\prime} 7\right)$, it was used to extract the photometry. The PSF-subtracted images, where the afterglow had been removed, were given as input to SExtractor. We used the same detection image for the various filters in order to measure the flux in an identical aperture. Our results are given in Table 2. Note that the galaxy is very red with $R-K=4.3$.

Due to the faintness of the $\mathrm{OA}$ at $\Delta t \approx 6.65$ days, and to the fact that the seeing was comparable to the separation between the OA and J1213.1-2054.8, it was not possible to perform simple PSF photometry on the OA. Instead we proceeded in the following way. We calculated the OA position relative to three nearby stars in the first VLT epoch, where the OA was much brighter than J1213.1-2054.8. We then created a PSF-subtracted image ( $\Delta t \approx 6.65$ days) to remove the majority of the J1213.1-2054.8 light. At the position of the OA we finally subtracted the PSF by varying its magnitude until the residuals had been adequately removed.

\subsection{Light curve}

Our optical/NIR afterglow light curve is presented in Fig. 3. As the $R$-band was better sampled than the other filters we analyse it in detail below. We fit the light curve with a broken powerlaw (with indices $\alpha_{1}$ and $\alpha_{2}$ before and after the break, respectively) in three different ways: $(i)$ including all data points in the fit; (ii) excluding the bump around $\Delta t \approx 1.8$ days, assuming it is short-lived and consisting of three points; (iii) excluding the bump, assuming it is long-lived and sampled by six points, and fixing $\alpha_{2}$ at a value obtained from the SED fitting, where $\alpha_{2}=-p$ and $p$ is the electron energy power-law index (see Sect. 5.1). The results of the fitting are listed in Table 3.

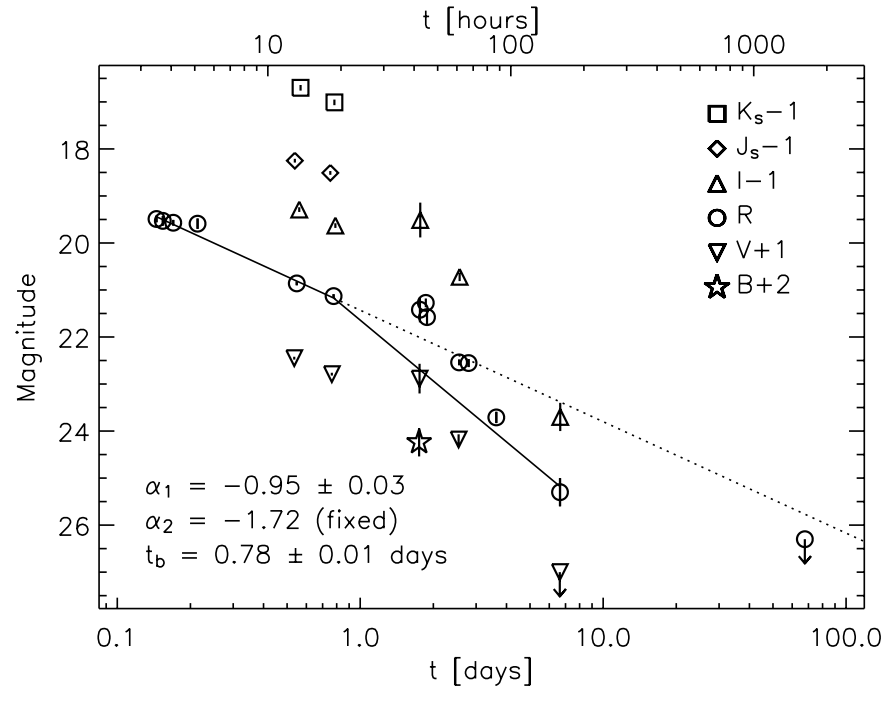

Fig. 3. The optical/NIR light curve of the afterglow based on the measurements given in Table 1. The dotted line is an extrapolation of the straight line fit through the early $R$-band data. The power-law indices and time of the break refer to the $R$-band fit.

Due to the bump in the light curve, $(i)$ is formally rejected with high significance. A better fit is obtained in (ii) by omitting the three points clustered around $\Delta t \approx 1.8$ days. On the other hand, in this case $\Delta \alpha \equiv \alpha_{1}-\alpha_{2}=2.47 \pm 0.44$, a value too high to be consistent with any of the fireball model predictions (see discussion in Sect. 5.1). The best fit is acquired in (iii), indicating there is an increase in the OA flux above the extrapolated power-law, lasting for approximately 2-3 days. We note the late-time behaviour of the light curve is consistent with the observations of Khamitov et al. (2003) who find $R=23.5 \pm 0.5$ at $\Delta t=3.43$ days.

The other filters display a similar initial decay index, with $\alpha_{V} \sim \alpha_{I} \sim \alpha_{K \mathrm{~s}} \sim-0.9$ and $\alpha_{J_{\mathrm{s}}} \sim-0.7$. In addition, the final $I$-band detection clearly implies that the light curve has steepened, with a similar late-time decay index as in the $R$-band. The upper limit in $V$ supports the same conclusion for that filter. The bump is also present in the VI filters as indicated by the points obtained with the $1.0-\mathrm{m}$ telescope. The data points covering the bump are close enough in time compared to the bump time scale in order to compute a reasonable estimate of the $V-R$ and $R-I$ colours. These are consistent with the same colours calculated from two previous epochs, $\Delta t=0.548$ days and $\Delta t=0.777$ days (see Sect. 3.3). In conclusion, the light curve displays an achromatic behaviour with a bump lasting for a couple of days.

\subsection{Spectral energy distribution of the afterglow}

Our multiband observations of GRB 030429 allowed the construction of the SED at two epochs. We interpolated the magnitudes to common epochs $(\Delta t=0.548$ days and $\Delta t=0.777$ days), using the aforementioned power-law indices. We note that the flux from the host galaxy has not been subtracted. However, the host is faint enough $(R>26.3)$ as seen at $\Delta t \approx 67$ days, that it should contribute $<1 \%$ of the flux 


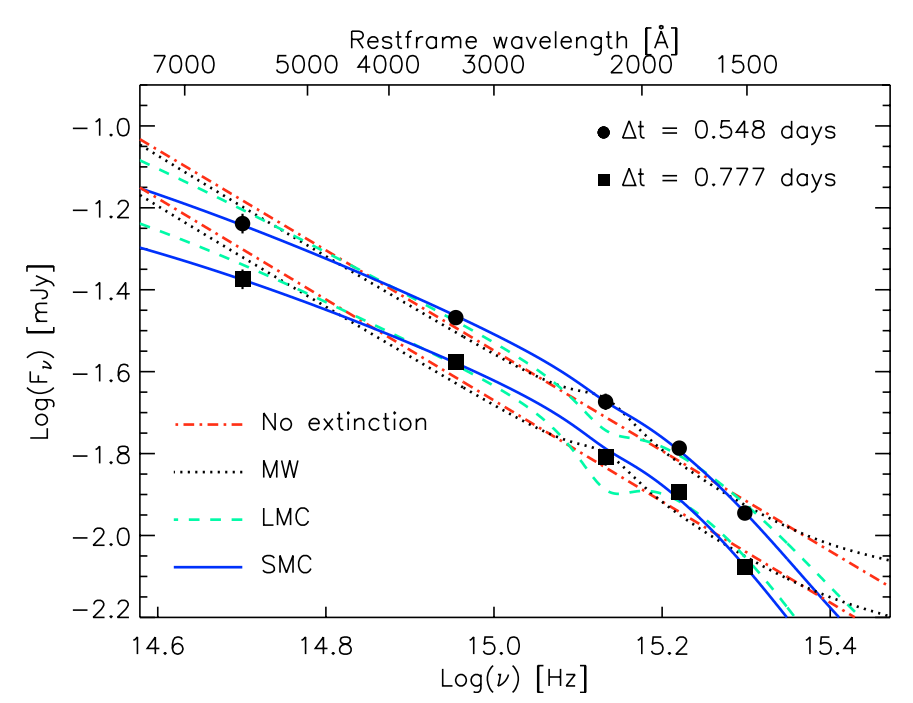

Fig. 4. The spectral energy distribution (SED) of the GRB 030429 afterglow at $\Delta t=0.548$ days (filled circles) and $\Delta t=0.777$ days (filled squares). The data points are based on our $K_{\mathrm{s}} J_{\mathrm{s}} I R V$ measurements. The abscissa displays the frequency/wavelength in the GRB restframe, assuming $z=2.658$ (see Sect. 4.2). At both epochs the SED is best fit by an underlying $\beta \simeq-0.36$ power-law with an SMC extinction law with $A_{V} \simeq 0.34$.

at the epochs we are exploring. The SED was constructed as explained in Fynbo et al. (2001a). The result is shown in Fig. 4, where we have corrected the observed data points for foreground (Galactic) extinction using the reddening maps of Schlegel et al. (1998), giving $E(B-V)=0.062$ at that position on the sky.

In order to quantify the effects of extinction we assume that the intrinsic spectrum of the afterglow is a power-law, and fit the function $F_{v} \propto v^{\beta} \times 10^{-0.4 A_{v}}$ to the observed SED, where $A_{v}$ is the extragalactic extinction along the line-of-sight to the burst. The dependence of $A_{v}$ on $v$ has been parameterised in terms of the restframe $A_{V}$ following the three extinction laws given by Pei (1992) for the Milky Way (MW), Large Magellanic Cloud (LMC) and Small Magellanic Cloud (SMC). For the assumed extinction law, the fit provides $\beta$ and $A_{V}$ simultaneously (see e.g. Jensen et al. 2001; Fynbo et al. 2001a; Holland et al. 2003; Jakobsson et al. 2003; Hjorth et al. 2003a; Vreeswijk et al. 2004). For comparison purposes we also considered the unextincted case, a pure power-law spectrum given by $F_{v} \propto v^{\beta}$.

The fits are shown in Fig. 4, while the parameters of the fits for both epochs are given in Table 4. The SMC fit is fully consistent with the data from both epochs, while other fits are formally rejected with high significance. For the redshift of GRB 030429 the interstellar extinction bump at $2175 \AA$ falls into the observed $I$-band. As expected by the lack of this absorption bump in Fig. 4, the MW and LMC extinction are completely inconsistent with the data. The absence of the $2175 \AA$ feature, often ascribed to graphite grains and ubiquitous in the spectra of sight lines through the Galactic diffuse interstellar medium, suggests that the GRB host galaxy has lower metallicity and dust content than the MW and the LMC. In conclusion, the featureless SMC extinction law provides the best fit at
Table 4. The results of fitting different extinction laws to our GRB $030429 K_{\mathrm{s}} J_{\mathrm{s}} I R V$ afterglow observations. We note that $\chi_{\text {d.o.f. }}^{2}=$ $\chi^{2} /$ degree of freedom, is the reduced $\chi^{2}$ of the fit.

\begin{tabular}{lcccc}
\hline \hline Fitting function & $\chi_{\text {d.o.f. }}^{2}$ & d.o.f. & $\beta$ & $A_{V}$ \\
\hline \multicolumn{1}{c}{$\Delta t=0.548$ days: } & & & & \\
No extinction & 7.45 & 3 & $-1.22 \pm 0.04$ & 0 \\
MW & 7.84 & 2 & $-1.37 \pm 0.07$ & $<0$ \\
LMC & 8.86 & 2 & $-0.70 \pm 0.24$ & $0.30 \pm 0.13$ \\
SMC & 0.14 & 2 & $-0.44 \pm 0.17$ & $0.30 \pm 0.06$ \\
$\quad \Delta t=0.777$ days: & & & & \\
No extinction & 12.01 & 3 & $-1.22 \pm 0.04$ & 0 \\
MW & 15.50 & 2 & $-1.35 \pm 0.07$ & $<0$ \\
LMC & 11.08 & 2 & $-0.31 \pm 0.24$ & $0.52 \pm 0.14$ \\
SMC & 1.17 & 2 & $-0.27 \pm 0.17$ & $0.37 \pm 0.06$ \\
\hline
\end{tabular}

both epochs, with an average extinction of $A_{V}=0.34 \pm 0.04$ and a spectral index of $\beta=-0.36 \pm 0.12$. This is the most accurate $A_{V}$ determination obtained so far for a GRB host galaxy. We note that our estimation of $A_{V}$ is not severely limited by the lack of $B$ - and $U$-band data since the flux observed in those bands would be attenuated by the Ly $\alpha$ forest. At $z=2.658$ (see Sect. 4.2) the effective GRB restframe wavelength of these filters is indeed located below $1215 \AA$.

\section{Spectroscopy}

\subsection{Reduction and spectral extraction}

The data reduction was performed with standard techniques for bias and flat-field corrections. The individual spectra were coadded, and the resulting combined science frame was sky subtracted in the following way. In the science frame, regions on both sides of the spectra were filtered along the spatial axis with a $1 \times 13$ pixels median filter in order to remove cosmic ray hits. These regions were averaged to produce a 1D sky spectrum. The mean sky spectrum was expanded to a 2D spectrum by duplicating the 1D sky spectrum, and this was subtracted from the unfiltered science frame.

The spectra were optimally extracted using the code described in Møller (2000). The output of the code is the extracted 1D spectra and the 2D residual spectra. The spectral PSFs of the OA and J1213.1-2054.8 overlap in our 2D spectrum, so in order to separate the two contributions we employed an iterative procedure: (i) extract and remove the OA spectrum; (ii) extract the $2 \mathrm{D}$ spectrum of the neighbouring galaxy and subtract it from the original spectrum containing both components; (iii) extract the 2D spectrum of the afterglow and subtract it from the original spectrum containing both components. After four iterations of (ii) and (iii) a stable solution was found.

The resulting spectra were wavelength-calibrated using the dispcor task in $I R A F^{2}$, with an error in the wavelength

\footnotetext{
${ }^{2} I R A F$ is distributed by the National Optical Astronomy Observatories, which are operated by the Association of Universities for Research in Astronomy, Inc., under cooperative agreement with the National Science Foundation.
} 

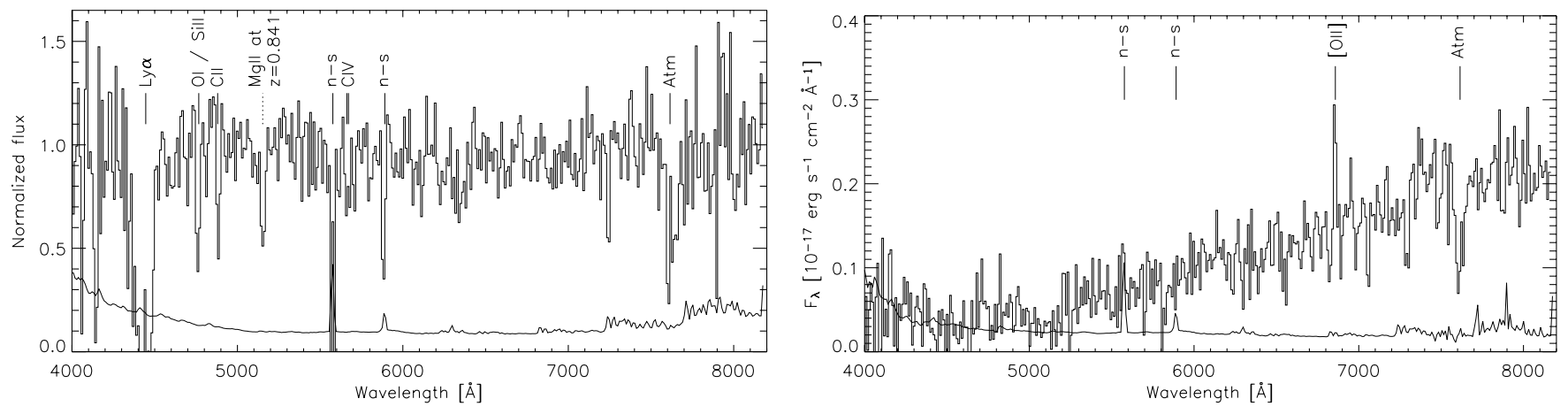

Fig. 5. Left: the normalized OA spectrum 2.77 days from the onset of the burst. We detect five absorption lines in the spectrum. The identifications are presented in Table 5. Right: the spectrum of J1213.1-2054.8. We detect one emission line in the spectrum. In both panels the spectra have been rebinned to match the size of a resolution element (10 ̊), and the lower curve is the Poisson error spectrum. Strong residuals from night-sky emission lines are marked with "n-s".

Table 5. Features detected in both the OA and J1213.1-2054.8 spectra. The columns show the line identification, the restframe wavelength, the observed wavelength, the restframe equivalent width (negative for absorption lines) and the inferred redshift of the lines, respectively.

\begin{tabular}{lcccc}
\hline \hline Line & $\begin{array}{c}\lambda_{\text {rest }} \\
{[\AA]}\end{array}$ & $\begin{array}{c}\lambda_{\text {obs }} \\
{[\AA]}\end{array}$ & $\begin{array}{c}W_{\text {rest }} \\
{[\AA]}\end{array}$ & $z$ \\
\hline \multicolumn{1}{c}{ Afterglow: } & & & & \\
Ly $\alpha$ & - & - & $42.1 \pm 2.9$ & - \\
O I / Si II & 1303.3 & 4764.8 & $2.2 \pm 0.4$ & 2.6559 \\
C II & 1334.5 & 4885.7 & $2.5 \pm 0.5$ & 2.6610 \\
C IV (doublet) & - & - & $1.4 \pm 0.4$ & - \\
Mg II (doublet) & - & - & $3.3 \pm 0.4$ & 0.8418 \\
$\quad$ J1213.1-2054.8: & & & & \\
[O II $]$ & 3727.1 & 6858.0 & $-6.8 \pm 0.8$ & 0.8400 \\
\hline
\end{tabular}

solution of the order of $0.3 \AA$ (only $3 \%$ of our spectral resolution). Flux calibration was performed using an observation of the standard star LTT7987. Finally, the spectra were rescaled to match the $V$-band magnitude at the same epoch. In Fig. 5 we show the spectra of both the OA and J1213.1-2054.8.

\subsection{Redshift}

We identify three absorption lines at $>5 \sigma$ in the spectrum of the OA. We use two of them, namely the narrow lines O I/Si II and C II, to calculate the redshift of the GRB, placing it at $z=2.658 \pm 0.004$. Armed with this redshift we searched for additional lines at $>3 \sigma$. At the expected position of the C IV doublet we find a likely candidate, although at our spectral resolution we are unable to resolve it. In the spectrum of J1213.1-2054.8 we detect one emission line, which we tentatively identify as [O II] at a redshift of $z=0.84$. Checking this redshift against the OA spectrum, we identify an absorption line at the location of the Mg II doublet. The redshift of this blended $\mathrm{Mg}$ II line is based on a minimum $\chi^{2}$ fitting of the expected line profile. We conclude that the redshift of the nearby galaxy is $z=0.841 \pm 0.001$. The detected lines and their characteristics are listed in Table 5 . These results rule out J1213.1-2054.8 as being the GRB 030429 host galaxy.

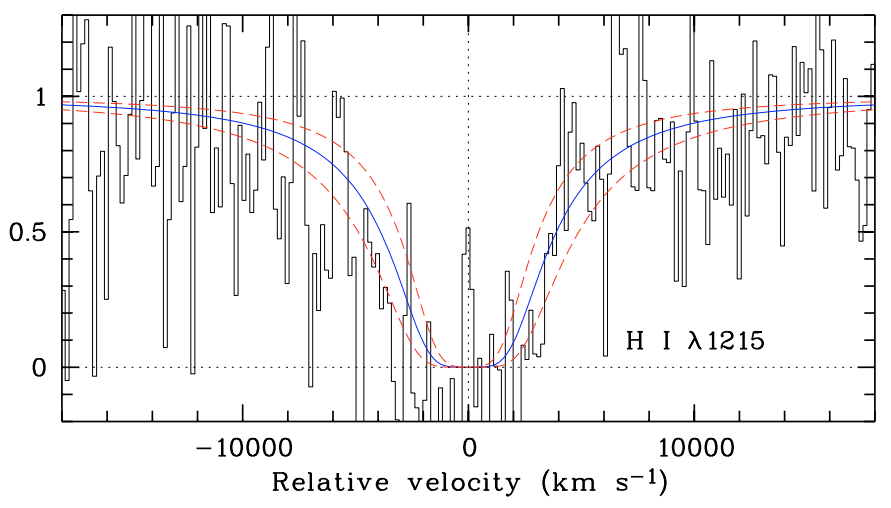

Fig. 6. The normalized OA spectrum centred on the Ly $\alpha$ absorption line. A neutral hydrogen column density fit to the damped Ly $\alpha$ line is shown with a solid line, while $1 \sigma$ errors are shown with dashed lines.

\subsection{The damped Ly $\alpha$ absorption line}

Absorption systems with neutral hydrogen column density larger than $2 \times 10^{20} \mathrm{~cm}^{-2}$ are referred to as damped Ly $\alpha$ absorbers (DLAs) as they display strong Ly $\alpha$ damping wings even in low/medium resolution spectra. These are normally detected in QSO spectra but so far four GRB afterglows have shown evidence for a high column density DLA system (see e.g. Fig. 4 in Vreeswijk et al. 2004). Almost all of the neutral gas in the high- $z$ Universe is located in the DLAs and they represent a major fraction of the baryon reservoir out of which stars at the present epoch formed (e.g. Storrie-Lombardi \& Wolfe 2000, and references therein).

In Fig. 6 we plot the normalized OA spectral region around Ly $\alpha$. Overplotted is a fit to the strong Ly $\alpha$ absorption line yielding $\log N(\mathrm{HI})=21.6 \pm 0.2$, within the range of previously known GRB-DLAs $(21.2 \leq \log N(\mathrm{HI}) \leq 21.9)$. The redshift deduced in the previous subsection, $z=2.658$, was imposed on the fit. Assuming $R_{V}=2.91$ and using $A_{V}=0.34 \pm 0.04$ from the best fit extinction law (SMC), the ratio between the neutral hydrogen column density and the reddening in the GRB 030429 host is $N(\mathrm{HI}) / E(B-V)=(34 \pm 16) \times 10^{21} \mathrm{~cm}^{-2} \mathrm{mag}^{-1}$. We note that this is a lower limit as $\mathrm{J} 1213.1-2054.8$ could contribute to the measured $A_{V}$. This value is consistent with that of the 
Table 6. Computation of the closure relation, $\left|\alpha_{1}\right|+b|\beta|+c$, for two afterglow models. A favourable model will have a value of zero for the closure relation. The ISM and wind models are for isotropic expansion in a homogeneous and wind-stratified environment, respectively. The electron energy power-law index, $p$, is obtained in two ways, from $\beta$ and $\alpha_{1}$. In addition, $\Delta \alpha$ is calculated from the resulting $p$ in both cases. The numbers in the table are based on case (iii) in Table 3.

\begin{tabular}{ccccccc}
\hline \hline Model & $v_{\mathrm{c}}$ & Closure & $p(\beta)$ & $\Delta \alpha$ & $p\left(\alpha_{1}\right)$ & $\Delta \alpha$ \\
\hline ISM & $v_{\mathrm{c}}>v_{\mathrm{o}}$ & $0.41 \pm 0.18$ & $1-2 \beta=1.72 \pm 0.24$ & $(p+3) / 4=1.18 \pm 0.06$ & $\left(4\left|\alpha_{1}\right|+3\right) / 3=2.27 \pm 0.04$ & $1.32 \pm 0.01$ \\
ISM & $v_{\mathrm{c}}<v_{\mathrm{o}}$ & - & $-2 \beta=0.72 \pm 0.24$ & - & - & - \\
Wind & $v_{\mathrm{c}}>v_{\mathrm{o}}$ & $-0.09 \pm 0.18$ & $1-2 \beta=1.72 \pm 0.24$ & $(p+1) / 4=0.68 \pm 0.06$ & $\left(4\left|\alpha_{1}\right|+1\right) / 3=1.60 \pm 0.04$ & $0.65 \pm 0.01$ \\
Wind & $v_{\mathrm{c}}<v_{\mathrm{o}}$ & - & $-2 \beta=0.72 \pm 0.24$ & - & - & - \\
\hline
\end{tabular}

SMC, $(44 \pm 7) \times 10^{21} \mathrm{~cm}^{-2} \mathrm{mag}^{-1}$, and similar to those found for other GRB absorbers (see Table 3 in Hjorth et al. 2003a).

There is an indication of $\operatorname{Ly} \alpha$ emission in the centre of the trough, but it is not statistically significant $(\$ 2 \sigma)$. The flux of the line, if real, is approximately $2.5 \times 10^{-18} \mathrm{erg} \mathrm{s}^{-1} \mathrm{~cm}^{-2}$. In our assumed cosmology this corresponds to a luminosity of $1.5 \times 10^{41} \mathrm{erg} \mathrm{s}^{-1}$. We can use this result to estimate the starformation rate (SFR), assuming the conversion from Ly $\alpha$ luminosity to the SFR of $10^{42} \mathrm{erg} \mathrm{s}^{-1}=1 M_{\odot} \mathrm{yr}^{-1}$ (Kennicutt 1998; Cowie \& Hu 1998). The Ly $\alpha$ SFR in the host of GRB 030429 is thus $\sim 0.15 M_{\odot} \mathrm{yr}^{-1}$. If confirmed this will strengthen the conclusion of Fynbo et al. (2003b) that Ly $\alpha$ emission is much more frequent among GRB host galaxies than among the LymanBreak galaxies at similar redshifts and that there could be a low metallicity preference for GRBs (see also Woosley \& MacFadyen 1999).

\section{Discussion}

\subsection{Comparison with afterglow models}

The nature of the ambient medium in which the GRB originated can be probed with the parameters $\alpha_{1}, \alpha_{2}$ and $\beta$. We consider two afterglow models: an isotropic expansion into a homogeneous medium (Sari et al. 1998), and an isotropic expansion into a wind-stratified medium (Chevalier \& Li 1999). We use the closure relation introduced by Price et al. (2002) in order to differentiate between the models. In our notation $\left|\alpha_{1}\right|+b|\beta|+c=0$, where the values of $b$ and $c$ depend on the location of the cooling frequency, $v_{\mathrm{c}}$, relative to the optical/NIR bands, $v_{0}$, at the epoch of the observations.

In Table 6 we have used the average value of the spectral index, $\beta=-0.36 \pm 0.12$, to derive the electron energy powerlaw index, resulting in $p=-2 \beta=0.72 \pm 0.24$ if $v_{\mathrm{c}}<v_{\mathrm{o}}$ and $p=1-2 \beta=1.72 \pm 0.24$ if $v_{\mathrm{c}}>v_{\mathrm{o}}$. The former value is not considered relevant in afterglow models, and the accurate analytical spectral and temporal indices have not been derived in the literature for this case.

The ISM model is not favoured by the closure relation. We have used the results from case (iii) (see Sect. 3.2), but note that (i) and ( $i$ i) give a similar result as $\alpha_{1}$ does not vary significantly between all three cases.

Each spectral or temporal power-law index relates to a certain value of the electron energy power-law index, $p$, and the correct model should result in a similar $p$ for all indices. Assuming that the fireball model is valid, this simple fact excludes $(i)$ and $(i i)$. In these cases $p(\beta) \neq p\left(\alpha_{2}\right) \neq p\left(\alpha_{1}\right)$, and the predicted $\Delta \alpha$ is in addition approximately twice as small as the observed one.

In conclusion, only the wind $\left(v_{\mathrm{c}}>v_{\mathrm{o}}\right)$ model produces a closure relation consistent with zero. We have fixed $\alpha_{2}=$ $-p(\beta)$, case (iii), which results in an observed $\Delta \alpha=0.77 \pm$ 0.03 . This is marginally consistent with the predicted values listed in Table 6.

The value of $p$ as estimated from $\beta, p(\beta)=1.72 \pm 0.24$, is marginally consistent with $p=2$. We derived the closure relations for the flat spectrum case, $1<p<2$ (Dai \& Cheng 2001; Bhattacharya 2001), and found that although formally consistent with the observationally determined value of $p(\beta)$, none of the closures were consistent with zero. In addition, the predicted $\Delta \alpha$ values were quite far from that observed. This suggest that the $p \gtrsim 2$ case is a better representation of the data, although not perfect. This may be due to insufficient sampling of the light cure and its overall structure (e.g. bumps).

Accepting the above scenario, it is clear from Fig. 3 that there is a bright long-lived achromatic bump above the late-time light curve fit. Due to the sparse data sampling we cannot constrain the properties of this bump in detail, but it lasts for 2-3 days and seems to peak at $1.5 \mathrm{mag}$ above the fit. Similar undulations have been observed previously in light curves associated with GRBs: GRB 970508 (e.g. Galama et al. 1998; Sokolov et al. 1998), GRB 000301C (e.g. Sagar et al. 2000; Garnavich et al. 2000; Masetti et al. 2000; Berger et al. 2000; Jensen et al. 2001; Rhoads \& Fruchter 2001; Dai \& Lu 2001; Panaitescu 2001; Gaudi et al. 2001), GRB 000911 (Ramirez-Ruiz et al. 2002), GRB 011211 (e.g. Holland et al. 2002; Jakobsson et al. 2004), GRB 021004 (e.g. Lazzati et al. 2002; Bersier et al. 2003; Heyl \& Perna 2003; Holland et al. 2003; Mirabal et al. 2003; Fox et al. 2003) and GRB 030329 (e.g. Lipkin et al. 2004, and references therein).

Deviations from the common smooth power-law decay have been explained by a variable external density (e.g. Wang \& Loeb 2000), refreshed shocks from the inner engine (e.g. Rees \& Mészáros 1998), a non-uniform jet structure (e.g. Mészáros et al. 1998) or a microlensing event (e.g. Garnavich et al. 2000). Simultaneous multi-wavelength observations can help to determine which of the above scenarios is responsible for OA light curve variations (e.g. Nakar \& Piran 2003; Nakar et al. 2003; Jakobsson et al. 2004). Armed only with a sparse optical/NIR data set for GRB 030429, we are unable to distinguish between these possibilities. 

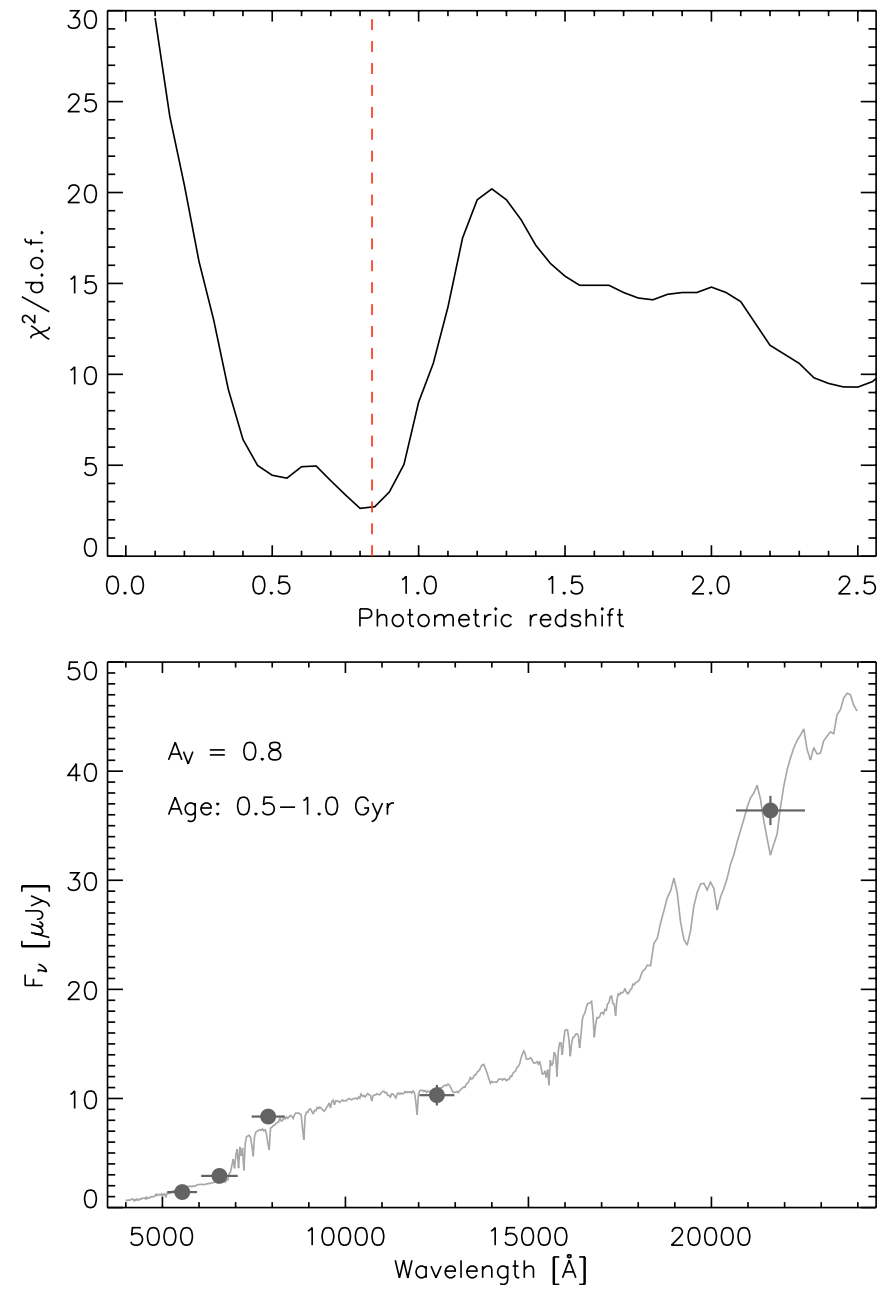

Fig. 7. Top: the reduced $\chi^{2}$ as a function of the photometric redshift of the nearby galaxy, obtained from hyperz. The best fit is obtained for $z=0.82$. The spectroscopic redshift of 0.841 is indicated by the vertical dashed line. Bottom: the best fit template galaxy spectrum along with the observed $V R I J_{\mathrm{s}} K_{\mathrm{s}} \mathrm{SED}$ based on the measurements given in Table 2 (filled circles).

\subsection{The intervening absorption galaxy}

In order to gain more information about J1213.1-2054.8 we used the photometric redshift code hyperz (Bolzonella et al. 2000). In the fitting process the programme uses standard $\chi^{2}$ minimisation, i.e. computing and minimising the deviations between the observed SED (see Table 2) of an object and template SEDs compared within the same photometric system. For J1213.1-2054.8 the best fit is obtained for a photometric redshift of $z=0.82 \pm 0.02$, in excellent agreement with the spectroscopic redshift of 0.841 that we previously determined (upper panel of Fig. 7).

The best fit template spectrum is displayed in Fig. 7 (lower panel). It corresponds to a fairly young stellar population, age between 0.5-1.0 Gyr, with a significant extinction of $A_{V}=0.8$. In addition, in J1213.1-2054.8 we have detected [O II] in emission (Table 5). All evidence is thus consistent with a star-forming galaxy. We calculate its absolute restframe $R$-band magnitude by transforming the observed $J$-band magnitude (Table 2) to AB magnitudes (Fukugita et al. 1995),
Table 7. GRBs whose spectra display an Mg II absorption system besides the one produced in the host galaxy. Here, $D$ is the impact parameter, the projected distance between the OA and the candidate $\mathrm{Mg}$ II absorber. The impact parameter is given in $\mathrm{kpc}$ where the absorber is spectroscopically confirmed.

\begin{tabular}{ccrc}
\hline \hline GRB & $z_{\mathrm{GRB}}$ & \multicolumn{1}{c}{$z_{\mathrm{abs}}$} & \multicolumn{1}{c}{$D$} \\
\hline 970508 & 0.835 & 0.768 & $\sim 5^{\prime \prime} .5$ \\
991216 & 1.022 & $0.770,0.803$ & $\sim 00^{\prime} 5, \sim 2^{\prime \prime} 0$ \\
010222 & 1.477 & $1.156,0.927$ & $\sim 2^{\prime \prime} 0, \sim 4{ }^{\prime \prime} 0$ \\
020405 & 0.691 & 0.472 & $13 \mathrm{kpc}\left(2^{\prime \prime} 0\right)$ \\
021004 & 2.335 & $1.381,1.604$ & $\sim 16^{\prime \prime}$ \\
030226 & 1.986 & 1.043 & $\sim 2^{\prime \prime} 5$ \\
030429 & 2.658 & 0.841 & $9 \mathrm{kpc}\left(1^{\prime \prime} .2\right)$ \\
\hline
\end{tabular}

applying the distance modulus and the $2.5 \log (1+z)$ term (see e.g. Appendix A in van Dokkum \& Franx 1996). This results in $M_{R}(\mathrm{AB})=-21.6$, a value located on the bright end of the luminosity function.

The galaxy is located 1'.2 from the line-of-sight to the OA, which corresponds to an impact parameter of $D=9 \mathrm{kpc}$ at $z=0.841$ in our adopted cosmology. $D$ is frequently interpreted as the lower limit of the radii of suspected counterparts of QSO absorption-selected galaxies. Guillemin \& Bergeron (1997) reported that galaxy counterparts of Mg II absorbers with equivalent widths larger than $0.6 \AA$ and $0.7<z<1.3$, all had $D>50 \mathrm{kpc}$. That result was consistent with the conclusions of Bergeron \& Boissé (1991), who examined a sample of $z \sim 0.5 \mathrm{Mg}$ II absorbers (see also Steidel 1995).

In every case, the possibility exists that the real absorber is very faint and/or hidden inside the glare of the QSO, thus overestimating the suspected counterpart radius. Indeed, more than half of present-day galaxies reside in groups (Eke et al. 2004), lending support to this hypothesis. The fact that many OAs are high- $z$ (Fig. 1) and transient phenomena has made it possible to search for faint/nearby absorbers. The OAs fade away completely within a few months, so it is only a matter of obtaining long exposures in order to get deep limits. GRB 030429 is the second burst in which a Mg II absorber with a small $D$ has been identified with imaging and spectroscopy. The GRB 020405 sight line also revealed a spectroscopically confirmed intervening $\mathrm{Mg}$ II absorber with an impact parameter of only $13 \mathrm{kpc}$ (Masetti et al. 2003).

In Table 7 we list all GRBs that have an Mg II absorption system along the line of sight in addition to that originating from the host galaxy. We have omitted bursts whose spectra display an Mg II absorption that most likely arises in a system physically interacting with the host (GRB 000926: Castro et al. 2003; GRB 020813: Barth et al. 2003). Of the seven bursts, two (GRB 020405 and GRB 030429) have a spectroscopically confirmed counterpart with a very small impact parameter of $D \sim 10 \mathrm{kpc}$. The host of GRB 991216 is located close to at least three nearby galaxies which are likely candidates for the two Mg II absorption systems (Vreeswijk 2002). Should that 
be the case, their impact parameters would correspond to only $4-15 \mathrm{kpc}$. The situation is similar for GRB 010222, where at least four nearby galaxies surround its host (e.g. Mirabal et al. 2002; Frail et al. 2002; Galama et al. 2003). Two galaxies are located 2.'5 away from GRB 030226, corresponding to $20 \mathrm{kpc}$ at $z=1.043$ (e.g. Price et al. 2003; Klose et al. 2004). A possible galaxy counterpart to the Mg II system in GRB 970508 is located 5'.5 away from the host, corresponding to $40 \mathrm{kpc}$ at $z=0.786$ (e.g. Metzger et al. 1997; Djorgovski et al. 1997; Pian et al. 1998; Sokolov et al. 1999; Fructher et al. 2000). For GRB 021004 a possible galaxy candidate at an impact parameter of $16^{\prime \prime}$, corresponding to $135 \mathrm{kpc}$ at $z=1.381$, has been observed in narrow-band imaging (Vreeswijk et al. 2003). The authors point out that a deeper study should be performed on the GRB 021004 field before a faint absorber at a low impact parameter can be ruled out.

A large fraction of possible OAs appears to have a faint absorber at small $D$. These results imply that some previous QSO absorption-selected galaxy counterpart identifications may be incorrect, with the real absorber being a nearby faint galaxy. This suggestion is supported by the observations of Falomo et al. (1997) and Watson et al. (2004), who find a likely counterpart to an Mg II absorption system in a BL Lac object at only $D=11 \mathrm{kpc}$. In addition, Ellison et al. (2004) analysed 27 intervening absorption systems in the spectra of a triply imaged QSO. They find that the most likely absorber coherence scale is $3 \mathrm{kpc}$, more than one order of magnitude smaller than the dimensions of $\mathrm{Mg}$ II absorbers deduced from the impact parameters.

\subsection{Strong gravitational lensing?}

With an angular separation of only 1'.2, J1213.1-2054.8 could affect the appearance of the OA via strong gravitational lensing (e.g. Narayan \& Bartelmann 1999; Grossman \& Nowak 1994). For that to occur the Einstein radius must be at least 1 ".2, which corresponds to a lens mass of $\gtrsim 5.4 \times 10^{11} M_{\odot}$. Using $M_{R}(\mathrm{AB})=-21.6$ gives a mass-to-light ratio of $\gtrsim 15 \Upsilon_{\odot}$ (where $\left.\Upsilon_{\odot} \equiv M_{\odot} / L_{\odot}\right)$. At $z=0.841$ we are only probing the innermost $9 \mathrm{kpc}$ of the galaxy. The aforementioned mass is roughly six times larger than for Milky Way-like galaxies within a similar radius. In addition, the critical surface mass density for this configuration is $0.43 \mathrm{~g} \mathrm{~cm}^{-2}$, a factor of three to four times that in normal galaxy lenses (see discussion regarding GRB 990123 in Andersen et al. 1999).

We also utilised the lensmodel package (Keeton 2001) to model J1213.1-2054.8 as a singular isothermal ellipsoid model with a flat rotation curve. Using our $R$-band image from $\Delta t=$ 67.6 days we constrained its ellipticity, $e \approx 0.16$, and position angle, $\phi \approx-49^{\circ}$. Knowing the distance between the lens and the OA, we varied the lens mass until the findsrc output resulted in a multiple image system. This occurred when the mass inside $9 \mathrm{kpc}$ reached $\gtrsim 3 \times 10^{11} M_{\odot}$, a value comparable to the one obtained above. In conclusion, the three independent methods discussed here virtually rule out that GRB 030429 was multiply imaged (with a time delay of roughly a month) by the nearby galaxy.

\section{Conclusions}

GRB 030429 occurred in a faint $(R>26.3)$ galaxy at a redshift of $z=2.658 \pm 0.004$. The derived neutral hydrogen column density is $\log N(\mathrm{HI})=21.6 \pm 0.2$, while the restframe reddening, obtained from the optical/NIR SED, is accurately measured to be $A_{V}=0.34 \pm 0.04$. We conclude that the high value of the ratio between column density and optical extinction is readily explained by a metal-poor environment, similar to that of the SMC. This is fully consistent with the favoured SMC-like extinction law obtained from the SED fit.

A nearby galaxy with a separation of only 1.2 is ruled out as the host, with $z=0.841 \pm 0.001$. This is the second time a galaxy adjacent to a GRB OA is found to be responsible for a Mg II absorption system in the OA spectrum. In both cases the impact parameter was small, $D \sim 10 \mathrm{kpc}$. At least three additional bursts have galaxy counterparts with similar small impact parameters that presumably account for their Mg II absorption systems. Thus, at least five out of seven GRB OAs displaying $\mathrm{Mg}$ II in absorption (see Table 7), have a nearby galaxy with $D \sim 10-20 \mathrm{kpc}$. This strongly indicates that previous identifications of many QSO absorption-selected galaxy counterparts are possibly incorrect. The remaining OA galaxy counterparts should be spectroscopically confirmed before a firmer conclusion can be drawn.

Within the framework of the afterglow synchrotron model (Sari et al. 1998), the GRB 030429 early-time decay index, $\alpha_{1}$, and spectral index, $\beta$, indicate that a jet $\left(v_{\mathrm{c}}>v_{\mathrm{o}}\right)$ expanding into a wind medium provides the most likely scenario for the data. The late-time decay index, $\alpha_{2}$, has to produce an electron energy power-law index, $p$, equivalent to that calculated from $\beta$. This suggests that a bright long-lived bump is present in the GRB 030429 light curve. We emphasise that densely sampled GRB light curves are of the utmost importance in order to observe these variations; without the $\Delta t \approx 1.8$ days GRB 030429 observations it would have been difficult to detect the flux increment above the interpolated power-law.

Note added in proof: After the submission of this paper, GRB 030429 was classified ${ }^{3}$ as an X-ray flash (XRF). Together with the unambiguous OA redshift determination (see Sect. 4.2), this makes it the first XRF with a spectroscopically confirmed absorption redshift. We note that within the uncertainties the burst is consistent with being an X-ray rich GRB. The most probable reason for this burst being classified as an $\mathrm{XRF}$ is a combination of its fairly low intrinsic $E_{\text {peak }}$, and the relatively high redshift of the burst (resulting in an observed $E_{\text {peak }} \approx 35 \mathrm{keV}$ ).

Acknowledgements. We thank the anonymous referee for a very positive and constructive report. We are grateful to Roland Vanderspek for providing us with various GRB 030429 properties derived from analysis of HETE data. P.J., G.B. and E.H.G. acknowledge support from a special grant from the Icelandic Research Council. J.P.U.F. and K.P. acknowledge support from the Carlsberg foundation. This work was supported by the Danish Natural Science Research Council (SNF). The authors acknowledge benefits from collaboration within the EU FP5 Research Training Network "Gamma-Ray Bursts: An Enigma and a Tool".

\footnotetext{
${ }^{3}$ http://space.mit.edu/HETE/Bursts/Data/
} 


\section{References}

Andersen, M. I., Castro-Tirado, A. J., Hjorth, J., et al. 1999, Science, 283,2075

Barth, A. J., Sari, R., Cohen, M. H., et al. 2003, ApJ, 584, L47

Berger, E., \& Frail, D. A. 2003, GCN\#2197

Berger, E., Sari, R., Frail, D. A., et al. 2000, ApJ, 545, 56

Bergeron, J., \& Boissé, P. 1991, A\&A, 243, 344

Bersier, D., Stanek, K. Z., Winn, J. N., et al. 2003, ApJ, 584, L43

Bertin, E., \& Arnouts, S. 1996, A\&AS, 117, 393

Bhattacharya, D. 2001, BASI, 29, 107

Bolzonella, M., Miralles, J.-M., \& Pelló, R. 2000, A\&A, 363, 476

Castro, S., Galama, T. J., Harrison, F. A., et al. 2003, ApJ, 586, 128

Chevalier, R. A., \& Li, Z.-Y. 1999, ApJ, 520, L29

Cowie, L. L., \& Hu, E. M. 1998, AJ, 115, 1319

Dai, Z. G., \& Lu, T. 2001, A\&A, 367, 501

Dai, Z. G., \& Cheng, K. S. 2001, ApJ, 558, L109

Djorgovski, S. G., Metzger, M. R., Kulkarni, S. R., et al. 1997, Nature, 387,876

Doty, J., Vanderspek, R., Butler, N., et al. 2003, GCN\#2211

Eke, V. R., Baugh, C. M., Cole, S., et al. 2004, MNRAS, 348, 866

Ellison, S. L., Ibata, R., Pettini, M., et al. 2004, A\&A, 414, 79

Falomo, R., Kotilainen, J., Pursimo, T., et al. 1997, A\&A, 321, 374

Fox, D. W., Yost, S., Kulkarni, S. R., et al. 2003, Nature, 422, 284

Frail, D. A., Bertoldi, F., Moriarty-Schieven, G. H., et al. 2002, ApJ, 565,829

Fruchter, A. S., Pian, E., Gibbons, R., et al. 2000, ApJ, 545, 664

Fukugita, M., Shimasaku, K., \& Ichikawa, T. 1995, PASP, 107, 945

Fynbo, J. P. U., Gorosabel, J., Dall, T. H., et al. 2001a, A\&A, 373, 796

Fynbo, J. P. U., Gorosabel, J., Møller, P., et al. 2001b, The Optical Afterglow and Host Galaxy of GRB 000926, in Lighthouses of the Universe: The Most Luminous Celestial Objects and Their Use for Cosmology, ed. M. Gilfanov, R. Sunyaev, \& E. Churazov (Garching: Springer), 187

Fynbo, J. P. U., Hjorth, J., Klose, S., et al. 2003a, GCN\#2185

Fynbo, J. P. U., Jakobsson, P., Møller, P., et al. 2003b, A\&A, 406, L63

Galama, T. J., Groot, P. J., van Paradijs, J., et al. 1998, ApJ, 497, L13

Galama, T. J., Reichart, D., Brown, T. M., et al. 2003, ApJ, 587, 135

Garnavich, P. M., Loeb, A., \& Stanek, K. Z. 2000, ApJ, 544, L11

Gaudi, B. S., Granot, J., \& Loeb, A. 2001, ApJ, 561, 178

Gilmore, A., Kilmartin, P., \& Henden, A. 2003, GCN\#2184

Grossman, S. A., \& Nowak, M. A. 1994, ApJ, 435, 548

Guillemin, P., \& Bergeron, J. 1997, A\&A, 328, 499

Henden, A. 2003, GCN\#2214

Heyl, J. S., \& Perna, R. 2003, ApJ, 586, L13

Hjorth, J., Møller, P., Gorosabel, J., et al. 2003a, ApJ, 597, 699

Hjorth, J., Sollerman, J., Møller, P., et al. 2003b, Nature, 423, 847

Holland, S. T., Soszynski, I., Gladders, M. D., et al. 2002, AJ, 124, 639

Holland, S. T., Weidinger, M., Fynbo, J. P. U., et al. 2003, AJ, 125, 2291

Jakobsson, P., Hjorth, J., Fynbo, J. P. U., et al. 2003, A\&A, 408, 941

Jakobsson, P., Hjorth, J., Ramirez-Ruiz, E., et al. 2004, New Astron., 9, 435

Jensen, B. L., Fynbo, J. P. U., Gorosabel, J., et al. 2001, A\&A, 370, 909

Keeton, C. R. 2001, ApJ, submitted [arXiv: astro-ph/0102340]
Kennicutt, R. C. 1998, ARA\&A, 36, 189

Khamitov, I., Bikmaev, I., Parmaksizoglu, M., et al. 2003, GCN\#2208

Klose, S., Greiner, J., Rau, A., et al. 2004, AJ, in press [arXiv: astro-ph/0408041]

Landolt, A. U. 1992, AJ, 104, 340

Lazzati, D., Rossi, E., Covino, S., Ghisellini, G., \& Malesani, D. 2002, A\&A, 396, L5

Lipkin, Y. M., Ofek, E. O., Gal-Yam, A., et al. 2004, ApJ, 606, 381

Masetti, N., Bartolini, C., Bernabei, S., et al. 2000, A\&A, 359, L23

Masetti, N., Palazzi, E., Pian, E., et al. 2003, A\&A, 404, 465

Mészáros, P., Rees, M. J., \& Wijers, R. 1998, ApJ, 499, 301

Metzger, M. R., Djorgovski, S. G., Kulkarni, S. R., et al. 1997, Nature, 387,879

Mirabal, N., Halpern, J. P., Kulkarni, S. R., et al. 2002, ApJ, 578, 818

Mirabal, N., Halpern, J. P., Chornock, R., et al. 2003, ApJ, 595, 935

Møller, P. 2000, Messenger, 99, 31

Nakar, E., \& Piran, T. 2003, ApJ, 598, 400

Nakar, E., Piran, T., \& Granot, J. 2003, New Astron., 8, 495

Narayan, R., \& Bartelmann, M. 1999, Gravitational Lensing, in Formation of Structure in the Universe, ed. A. Dekel, \& J. P. Ostriker (University Press, Cambridge), 360

Panaitescu, A. 2001, ApJ, 556, 1002

Pei, Y. C. 1992, ApJ, 395, 130

Pian, E., Fruchter, A. S., Bergeron, L. E., et al. 1998, ApJ, 492, L103

Price, P. A., Berger, E., Reichart, D. E., et al. 2002, ApJ, 572, L51

Price, P. A., Fox, D. W., Djorgovski, S. G., et al. 2003, GCN\#1889

Ramirez-Ruiz, E., Dray, L. M., Madau, P., \& Tout, C. A. 2001, MNRAS, 327, 829

Rees, M. J., \& Mészáros, P. 1998, ApJ, 496, L1

Rhoads, J. E., \& Fruchter, A. S. 2001, ApJ, 546, 117

Sagar, R., Mohan, V., Pandey, S. B., et al. 2000, BASI, 28, 499

Sari, R., Piran, T., \& Narayan, R. 1998, ApJ, 497, L17

Schlegel, D. J., Finkbeiner, D. P., \& Davis, M. 1998, ApJ, 500, 525

Sokolov, V. V., Kopylov, A. I., Zharikov, S. V., et al. 1998, A\&A, 334, 117

Sokolov, V. V., Zharikov, S. V., Baryshev, Y. V., et al. 1999, A\&A, 344,43

Stanek, K. Z., Matheson, T., Garnavich, P. M., et al. 2003, ApJ, 591, L17

Steidel, C. C. 1995, The Nature and Evolution of Absorption-Selected Galaxies, in QSO Absorption Lines, ed. G. Meylan (New York: Springer), 139

Stetson, P. 1987, PASP, 99, 191

Stetson, P. 1994, PASP, 106, 250

Storrie-Lombardi, L. J., \& Wolfe, A. M. 2000, ApJ, 543, 552

van Dokkum, P. G., \& Franx, M. 1996, MNRAS, 281, 985

van Paradijs, J., Groot, P. J., Galama, T., et al. 1997, Nature, 386, 686

Vreeswijk, P. M. 2002, Ph.D. Thesis

Vreeswijk, P. M., Møller, P., \& Fynbo, J. P. U. 2003, A\&A, 409, L5

Vreeswijk, P. M., Ellison, S. L., Ledoux, C., et al. 2004, A\&A, 419, 927

Wang, X., \& Loeb, A. 2000, ApJ, 535, 788

Watson, D., McBreen, B., Hanlon, L., et al. 2004, A\&A, 418, 459

Weidinger, M., Fynbo, J. P. U., Hjorth, J., et al. 2003a, GCN\#2196

Weidinger, M., Fynbo, J. P. U., Hjorth, J., et al. 2003b, GCN\#2215

Woosley, S. E., \& MacFadyen, A. I. 1999, A\&AS, 138, 499 